\title{
Serological and Molecular Evaluation of Leishmania infantum Infection in Stray Cats in a Nonendemic Area in Northern Italy
}

\author{
Eva Spada, ${ }^{1}$ Daniela Proverbio, ${ }^{1}$ Antonella Migliazzo, ${ }^{2}$ Alessandra Della Pepa, \\ Roberta Perego, ${ }^{1}$ and Giada Bagnagatti De Giorgi ${ }^{1}$ \\ ${ }^{1}$ Dipartimento di Scienze Veterinarie per la Salute, la Produzione Animale e la Sicurezza Alimentare, \\ Università degli Studi di Milano, Via G. Celoria 10, 20133 Milano, Italy \\ ${ }^{2}$ Centro di Referenza Nazionale per le Leishmaniosi, Istituto Zooprofilattico Sperimentale della Sicilia, \\ Via R. Dicillo 4, 90129 Palermo, Italy
}

Correspondence should be addressed to Eva Spada; eva.spada@unimi.it

Received 8 May 2013; Accepted 5 June 2013

Academic Editors: M. Mahieu, P. Somboon, K. R. Trenholme, J. Venegas Hermosilla, and R. Zufferey

Copyright (C) 2013 Eva Spada et al. This is an open access article distributed under the Creative Commons Attribution License, which permits unrestricted use, distribution, and reproduction in any medium, provided the original work is properly cited.

\begin{abstract}
Infection by Leishmania species is increasing worldwide. It was hypothesized recently that cats act as a secondary reservoir for Leishmania infection. The aim of the present study was to assess the prevalence of Leishmania infantum antibodies and DNA in blood samples collected in a sample of stray cats in metropolitan area of Milan in northern Italy, which is a nonendemic area for leishmaniasis. An indirect immunofluorescence antibody test for L. infantum showed that 59 of 233 cats (25.3\%) were seroreactive, 38 samples (16.3\%) had antibody titers of $1: 40,15(6.4 \%)$ had antibody titers of $1: 80$, and $6(2.6 \%)$ had antibody titers of $1: 160$. Feline immunodeficiency virus (FIV) seropositive status was statistically associated with seroreactivity to $L$. infantum $(P=0.01)$ as shown by univariate and multivariate logistic regression $(P=0.0098 ; \mathrm{OR}=7.34)$. All blood samples that were tested using real-time PCR were negative for parasite DNA. These results were surprising, since no autochthonous human or canine cases of leishmaniasis have ever been reported in this region of northern Italy. It is possible that this high seroreactivity to L. infantum could be due to cross-reaction with antigens from other parasites. Additional studies that include parasite isolation are needed to clarify our findings on feline leishmaniasis in this region.
\end{abstract}

\section{Introduction}

Leishmaniasis in the Old World is caused by the protozoa Leishmania infantum. It is prevalent in countries in the Mediterranean basin, and dogs are the main reservoir of the parasite in that region [1]. In recent years, autochthonous cases of human and canine disease have been recorded at higher latitudes, namely, in Germany [2,3], The Netherlands [4], and North America [5]. Infections have also been reported in species other than dogs and humans, including horses [6] and cows [7]. There have been numerous reports of feline leishmaniasis (FeL), mostly in cats living in known endemic areas [8-10]; some of the cats had concurrent immunosuppressive infections [9-11]. In countries in southern Europe, where canine leishmaniasis (CanL) is endemic, serological investigations of feline populations have revealed seroprevalence rates ranging from less than $1 \%$ to more than
$60 \%$ [9-21]. Given the diffusion of Leishmania infection and the lack of information regarding infection rates in cats in the Milan metropolitan area in northern Italy, the aim of the present study was to assess the prevalence of leishmaniasis in a large representative sample of stray cats from this nonendemic area. A secondary aim was to analyze the results according to clinical, laboratory and infectious data.

\section{Materials and Methods}

2.1. Feline Population. During a 2-year period (January 2008 to January 2010), blood samples were collected from 233 European shorthair stray cats from urban colonies in Milan, northern Italy, during a trap-neuter-release (TNR) program that was approved by the local authority of the city council. The program was conducted as described previously [22]. 
2.2. Data Collection. The following data were recorded: sex $(n=233)$, age $(n=233)$, body condition score (BCS) $(n=215)$, area of colony of provenance, that is, one of the seven municipalities of Milan $(n=233)$, health status based on physical examination $(n=233)$, and dermatological evaluation ( $n=121$ ). Cats were classified as healthy or unhealthy depending on the clinical findings (Table 1).

2.3. Sample Collection. Whole blood samples were collected by cephalic or jugular venipuncture into tubes with EDTA anticoagulant for complete blood cell (CBC) count and polymerase chain reaction (PCR) testing and into empty tubes for serology. All samples used for serology and PCR were stored at $-20^{\circ} \mathrm{C}$ until use.

2.4. Hematological and Serological Examination. Within 24 hours of sample collection, a CBC count was performed on whole blood ( $n=127)$ using an ADVIA 120 System (Siemens Healthcare Diagnostics, Milan, Italy). Cats were categorized as having alterations in the CBC as shown in Table 1.

Serological assessment was performed to determine the presence of the following: antibodies to the feline immunodeficiency virus (FIV) relative to the gp40 and p24 FIV antigens, the feline leukemia virus (FeLV) p27 antigen (Snap FeLV/FIV Combo Plus Test, Idexx Laboratories, Hoofddorp, The Netherlands) ( $n=137$ ), and Toxoplasma gondii IgG antibodies (IFAT, Fuller Laboratories, Fullerton, CA, USA) $(n=79)$. The results of these serological tests have been already published [22] and were reanalyzed with the present results.

For various technical reasons, not all data were available for all 233 cats.

2.5. Indirect Immunofluorescence Antibody Test. The presence of anti-L. infantum antibodies was measured by an indirect immunofluorescence antibody test (IFAT) performed according to the recommendations of OIE [23] using MHOM/IT/80/IPT1 as a whole-parasite antigen fixed on multispot slides (Bio Merieux Spa, Florence, Italy) and fluorescently-labeled antifeline gamma globulin (Sigma Aldrich, Milan, Italy) as conjugate. Positive sera were diluted serially and tested to establish the maximum reaction titer, starting at a dilution of $1: 40$. Positive and negative controls were included on each slide.

2.6. PCR. L. infantum DNA was amplified from $200 \mu \mathrm{L}$ of whole blood by real-time PCR using the Illustra Blood genomicPrep Mini Spin kit (GE Healthcare, Milan, Italy) following the manufacturer's instructions. The target for amplification was a 116-bp fragment in the constant region of the kDNA minicircle of L. infantum. This is one of the kDNA minicircle families that is used to identify the Leishmania genus. The primers used were QLK2-UP $5^{\prime}$-GGCGTTCTGCGAAAACCG-3' and QLK2-DOWN $5^{\prime}$-AAAATGGCATTTTCGGGCC- $3^{\prime}$; the TaqManprobes were Q Leish Probe 2 and $5^{\prime}$-FAM TGGGTGCAGAAATCCCGTTCA-3' ${ }^{\prime}$-Black Hole.
2.7. Statistical Analysis. Univariate analysis of the categorical data was performed using the chi-square test or Fisher's exact test. Any parameters statistically linked to IFAT seroreactivity for $L$. infantum or to the presence of $L$. infantum DNA as detected by PCR were used in a logistic regression model to test for independent risk factors associated with the L. infantum positivity. Associations were considered statistically significant when $P<0.05$; both the $P$ value and odds ratio (OR) are reported. Data were analyzed using MedCalc Software (version 12.3.0; Mariakerke, Belgium).

\section{Results}

The characteristics of the feline study population are summarized in Table 1 . The serology test for L. infantum showed that $25.3 \%(59 / 233)$ of the cats had L. infantum seroreactivity, 38 (16.3\%) had antibody titers of 1:40, 15 (6.4\%) had titers of $1: 80$, and $6(2.6 \%)$ had antibody titers of $1: 160$. All blood samples tested using real-time PCR were negative for the presence of L. infantum DNA. Standard curve and amplification curve of real-time PCR were reported in Figures 1 and 2 , respectively.

No statistical association was found between seroreactivity to L. infantum and age, sex, BCS, municipality of provenance, clinical finding, dermatological findings or FeLV, and T. gondii serology. In contrast, in terms of CBC, neutrophilia was statistically associated with seroreactivity to L. infantum $(P=0.01)$ in univariate analysis, but this association was not confirmed using multivariate logistic regression $(P=$ 0.57). In terms of serology for the retrovirus, FIV seropositive status was statistically associated with seroreactivity to $L$. infantum $(P=0.01)$. This association was confirmed by multivariate logistic regression: $P=0.0098$ and $\mathrm{OR}=7.34$ (95\%CI $=1.96$ to 27.59$)$. The distribution of the parameters that were evaluated and compared in L. infantum seropositive and seronegative cats is shown in Table 1.

\section{Discussion}

This study is the first epidemiological investigation of feline Leishmania infection in the metropolitan area of Milan, which is a nonendemic area for leishmaniasis. We found seroreactivity to L. infantum by IFAT in 59 of the 233 $(25.3 \%)$ stray cats that we examined. These results were surprising, since no autochthonous human or canine cases of leishmaniasis have ever been reported in this region in northern Italy. In countries in southern Europe where leishmaniasis is endemic, serological investigations performed in feline populations using different techniques have revealed prevalence rates that range from less than $1 \%$ to more than $60 \%$ [9-21]. In particular, the seroprevalence in Italy ranges from $0.9 \%$ to $68 \%[9-11,13]$, in Spain from $3.7 \%$ to $60 \%$ [14-16], and in Portugal from $0.6 \%$ to $2.8 \%$ [18-20]. In Greece, the seroprevalence is 3.9\% [17] and in France it is $12.4 \%$ [12]. These results in L. infantum endemic geographical regions may reflect differences in the serological techniques used, in the cut-off values or positive thresholds and in the populations of cats that were tested. As here, previous 
TABLE 1: Characteristics of a population of stray cats in northern Italy and a comparison of characteristics in Leishmania infantum seropositive versus seronegative cats as determined using an indirect immunofluorescence antibody test.

\begin{tabular}{|c|c|c|c|c|c|}
\hline Factor & Category & $\begin{array}{c}\text { Total } \\
\text { population }\end{array}$ & $\begin{array}{c}\text { Sero } \\
\text { positive }\end{array}$ & $\begin{array}{c}\text { Sero } \\
\text { negative }\end{array}$ & Univariate $P$-value \\
\hline \multirow{2}{*}{ Age } & Young ( $\leq 6$ months) & $106(45.5 \%)$ & $24(40.7 \%)$ & $82(47.1 \%)$ & \multirow{2}{*}{0.4788} \\
\hline & Adult (>6 months) & $127(54.5 \%)$ & $35(59.3 \%)$ & $92(52.9 \%)$ & \\
\hline \multirow{2}{*}{ Sex } & Female & $153(65.7 \%)$ & $38(64.4 \%)$ & $115(66.1 \%)$ & \multirow{2}{*}{0.9387} \\
\hline & Male & $80(34.3 \%)$ & $21(35.6 \%)$ & $59(33.9 \%)$ & \\
\hline \multirow{2}{*}{ BCS } & Scarce $(\leq 3 / 9)$ & $19(8.8 \%)$ & $4(7.4 \%)$ & $15(9.3 \%)$ & \multirow{2}{*}{0.8802} \\
\hline & Good $(\geq 4 / 9)$ & $196(91.2 \%)$ & $50(92.6 \%)$ & $146(90.7 \%)$ & \\
\hline \multirow{7}{*}{ Colony of origin } & Zone 2 & $11(4.7 \%)$ & $2(3.4 \%)$ & $9(5.2 \%)$ & \multirow{7}{*}{0.0825} \\
\hline & Zone 4 & $95(40.8 \%)$ & $18(30.5 \%)$ & $77(44.2 \%)$ & \\
\hline & Zone 5 & $9(3.9 \%)$ & $0(0.0 \%)$ & $9(5.2 \%)$ & \\
\hline & Zone 6 & $23(9.9 \%)$ & $8(13.6 \%)$ & $15(8.6 \%)$ & \\
\hline & Zone 7 & $53(22.7 \%)$ & $17(28.8 \%)$ & $36(20.7 \%)$ & \\
\hline & Zone 8 & $21(9.0 \%)$ & $5(8.5 \%)$ & $16(9.2 \%)$ & \\
\hline & Zone 9 & $21(9.0 \%)$ & $9(15.2 \%)$ & $12(6.9 \%)$ & \\
\hline \multirow{7}{*}{ Clinical examination } & Healthy & $49(21.0 \%)$ & $12(20.3 \%)$ & $37(21.3 \%)$ & \multirow{2}{*}{0.9728} \\
\hline & Unhealthy & $184(79.0 \%)$ & $47(79.7 \%)$ & $137(78.7 \%)$ & \\
\hline & Stomatitis & $92(39.5 \%)$ & $17(28.8 \%)$ & $75(43.1 \%)$ & 0.0740 \\
\hline & Ocular discharge & $35(15.0 \%)$ & $10(16.9 \%)$ & $25(14.4 \%)$ & 0.7881 \\
\hline & Nasal discharge & $21(9.0 \%)$ & $5(8.5 \%)$ & $16(9.2 \%)$ & 0.9236 \\
\hline & Pale mucous membranes & $12(5.2 \%)$ & $4(6.8 \%)$ & $8(4.6 \%)$ & 0.7532 \\
\hline & Lymphadenomegaly & $117(50.2 \%)$ & $30(50.8 \%)$ & $87(50 \%)$ & 0.9696 \\
\hline \multirow{8}{*}{ Dermatological examination } & Absence of lesions & $83(68.6 \%)$ & $17(54.8 \%)$ & $66(73.3 \%)$ & \multirow{2}{*}{0.0912} \\
\hline & Presence of lesions & $38(31.4 \%)$ & $14(45.2 \%)$ & $24(26.7 \%)$ & \\
\hline & Crusted dermatitis & $22(18.2 \%)$ & $7(22.6 \%)$ & $15(16.7 \%)$ & 0.6410 \\
\hline & Scaling & $5(4.1 \%)$ & $1(3.2 \%)$ & $4(4.4 \%)$ & 0.8188 \\
\hline & Nodular dermatitis & $3(2.5 \%)$ & $2(6.5 \%)$ & $1(1.1 \%)$ & 0.3273 \\
\hline & Alopecia & $18(14.9 \%)$ & $8(25.8 \%)$ & $10(11.1 \%)$ & 0.0910 \\
\hline & Ectoparasites & $27(22.3 \%)$ & $6(22.2 \%)$ & $21(77.8 \%)$ & 0.8346 \\
\hline & Dermatophytosis & $9(7.4 \%)$ & $1(3.2 \%)$ & $8(8.9 \%)$ & 0.5225 \\
\hline \multirow{14}{*}{$\mathrm{CBC}$ results } & Absence of anemia & $29(22.8 \%)$ & $5(16.7 \%)$ & $24(24.7 \%)$ & \multirow{2}{*}{0.5015} \\
\hline & Presence of anemia & $98(77.2 \%)$ & $25(83.3 \%)$ & $73(75.3 \%)$ & \\
\hline & Decreased Ht & $97(76.4 \%)$ & $25(83.3 \%)$ & $72(74.2 \%)$ & 0.4352 \\
\hline & Decreased $\mathrm{Hb}$ & $23(18.1 \%)$ & $7(23.3 \%)$ & $16(16.5 \%)$ & 0.5627 \\
\hline & Decreased RBC & $41(32.3 \%)$ & $11(36.7 \%)$ & $30(30.9 \%)$ & 0.7158 \\
\hline & Thrombocytopenia & $10(7.9 \%)$ & $2(7.7 \%)$ & $8(8.2 \%)$ & 0.9149 \\
\hline & Leukocytosis & $5(3.9 \%)$ & $2(6.7 \%)$ & $3(3.1 \%)$ & 0.7319 \\
\hline & Leukopenia & $15(11.8 \%)$ & $3(10.0 \%)$ & $12(12.4 \%)$ & 0.9776 \\
\hline & Neutrophilia & $15(11.8 \%)$ & $8(26.7 \%)$ & $7(7.2 \%)$ & $\mathbf{0 . 0 1}(0.57)^{*}$ \\
\hline & Neutropenia & $2(1.6 \%)$ & $0(0.0 \%)$ & $2(2.1 \%)$ & 0.9631 \\
\hline & Lymphocytosis & $2(1.6 \%)$ & $0(0.0 \%)$ & $2(2.1 \%)$ & 0.9631 \\
\hline & Lymphopenia & $33(26.0 \%)$ & $12(40.0 \%)$ & $21(21.6 \%)$ & 0.0776 \\
\hline & Eosinophilia & $12(9.4 \%)$ & $4(13.3 \%)$ & $8(8.2 \%)$ & 0.6346 \\
\hline & Eosinopenia & $33(26.0 \%)$ & $9(30.0 \%)$ & $24(24.7 \%)$ & 0.7371 \\
\hline \multirow{2}{*}{ FIV status } & Positive & $12(8.8 \%)$ & $7(21.2 \%)$ & $5(4.8 \%)$ & $0.01(0.0098)^{*}$ \\
\hline & Negative & $125(91.2 \%)$ & $26(78.8 \%)$ & $99(95.2 \%)$ & $\mathrm{OR}=7.34^{*}(95 \%$ CI $1.96-27.59)$ \\
\hline
\end{tabular}


TABle 1: Continued.

\begin{tabular}{lccccc}
\hline Factor & Category & $\begin{array}{c}\text { Total } \\
\text { population }\end{array}$ & $\begin{array}{c}\text { Sero } \\
\text { positive }\end{array}$ & $\begin{array}{c}\text { Sero } \\
\text { negative }\end{array}$ & Univariate $P$-value \\
\hline FeLV status & Positive & $5(3.6 \%)$ & $0(0.0 \%)$ & $5(4.8 \%)$ & 0.453 \\
& Negative & $132(96.4 \%)$ & $33(100.0 \%)$ & $99(95.2 \%)$ & $17(31.5 \%)$ \\
\multirow{2}{*}{ T. gondii status } & Positive & $26(32.9 \%)$ & $9(36.0 \%)$ & $37(68.5)$ & 0.8886 \\
& Negative & $53(67.1 \%)$ & $16(64.0 \%)$ & \\
\hline
\end{tabular}

BCS: body condition score; CBC: complete blood count; Ht: hematocrit, Hb: hemoglobin, RBC: red blood cells, FIV: Feline immunodeficiency virus; FeLV: Feline Leukemia virus; OR: odds ratio; CI: confidence interval.

$P$-values in bold are statistically significant $(P<0.05)$.

${ }^{*}$ Results from multivariate logistic regression analysis.

epidemiological studies have used IFAT to detect antibodies to Leishmania spp. in cats. An important concern is that there is no standardized IFAT method for serological evaluation of antibodies to Leishmania spp. in cats; accordingly, there is no universally accepted antibody titer cut-off value that corresponds to active infection. Cut-off titers validated in dogs are used frequently for cats, but the immune response could be different in cats than in dogs.

None of the peripheral blood samples we examined using real-time PCR were positive for parasite DNA. PCR has been used previously by others, either alone or in combination with serology, as in our study, to assess the prevalence of feline Leishmania infection [9, 11, 14, 24, 25]. Blood is not the best specimen for PCR diagnosis of leishmaniasis. Specifically, PCR performed on canine blood has lower sensitivity, specificity, and positive and negative predictive values compared to PCR performed on canine lymph node aspirates [26], and this may be true for samples from cats as well. However, blood sampling is less invasive and is easy to perform, particularly for epidemiological studies involving numerous subjects, as in our survey.

Although dogs have been universally regarded as the domestic reservoir hosts of zoonotic visceral leishmaniasis caused by L. infantum, some researchers have hypothesized that cats may also act as a secondary reservoir host of leishmaniasis rather than simply as an accidental host $[9,14$, 15]. Differences in immune response, vector host preference, or innate resistance in cats to vector-borne diseases could account for the observed differences in the prevalence of infection in canine versus feline populations in endemic areas. Immunosuppressive agents, such as FIV or FeLV, or disease and stress, can induce immunological dysfunction and impair the cellular immune response. This allows active multiplication of the parasite and widespread visceral dissemination of the protozoa [27]. In our survey, FIV infection was statistically associated with seroreactivity to L. infantum by IFAT, and FIV-positive cats were 7.3 times more likely to be $L$. infantum seroreactive than FIV-negative cats $(P=$ $0.0098)$. This association has also been found in previous studies performed in endemic area of Southern Italy $[9,11]$.

Based on results from a recent survey, continental northern Italy is now focally endemic for leishmaniasis, but no sand-flies (vector) or autochthonous cases of human and canine leishmaniasis have been identified in Milan or its suburbs [28]. Cases of CanL are commonly diagnosed in the area where we performed our study, but the histories of the affected dogs always reveal that they have lived or travelled in areas that are endemic for CanL $[29,30]$. A canine epidemiological survey of 313 dogs in a public animal shelter that were tested for L. infantum by IFAT more than 10 years ago (20022003) in the urban area of Milan found a seroprevalence of $3.4 \%$ [31] Although the history of dogs in animal shelters is often unknown, some of these dogs may have come from areas that are endemic for L. infantum infection. In contrast, it is unlikely that all of the Leishmania seropositive cats found in our study population were infected in endemic areas. In the present study, the feline seroprevalence for $L$. infantum was much higher than the canine seroprevalence found 10 years previously in a canine population in an animal shelter in the same area. Notably, this area is still considered nonendemic for leishmaniasis. We speculate that the serology results for leishmaniasis in our survey may be an overestimation due to the possibility of IFAT crossreactivity between $L$. infantum and other pathogens. Crossreactivity with other pathogens is possible on some serologic tests, especially those that use a whole-parasite antigen, as we did here. There was no significant correlation between T. gondii positivity and L. infantum positivity in our study. This may suggest a lack of cross-reactivity with Toxoplasma parasites. New vector-borne parasites have been found that affect cats, such as Ehrlichia spp., Rickettsia felis, Anaplasma phagocytophilum, and Babesia spp. (according to the vectorborne disease ESCCAP guidelines) [32] that might be able to cross-react with Leishmania. This has been demonstrated in dogs in that IFAT cross-reactivity has been reported for L. infantum and Trypanosoma cruzi, Leishmania braziliensis, and Ehrlichia canis infection [33].

\section{Conclusions}

Our results demonstrate high levels of seroreactivity to L. infantum in cats in an area of northern Italy that has traditionally been considered to be free of leishmaniasis and nonendemic for this infection in dogs. Possible IFAT crossreactivity and a lack of a validated serological method for feline specie could explain our unexpectedly high seroprevalence. Additional studies that include parasite isolation are needed to clarify our findings on feline leishmaniasis in this geographic area. 


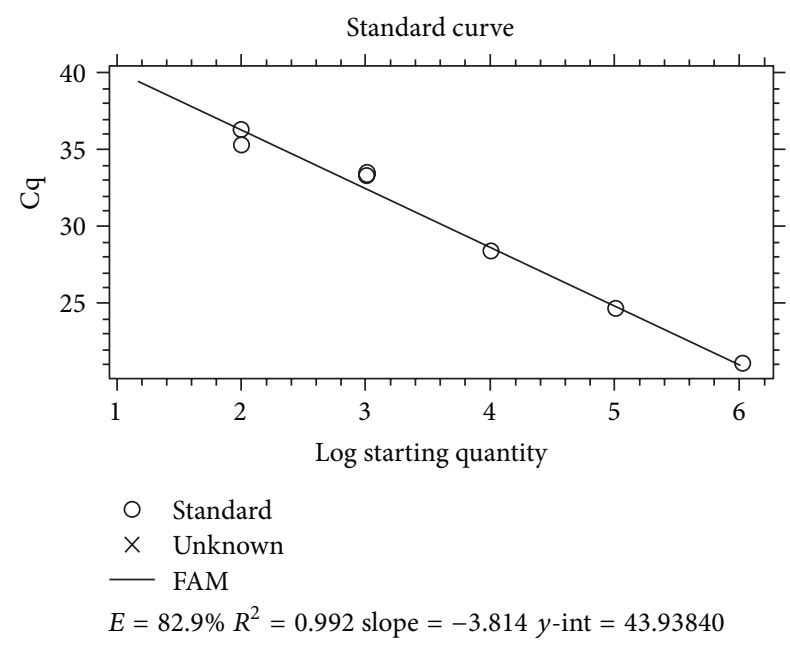

FIgURE 1: Standard curve in logarithmic scale.

Quantification

Step number: 4

Analysis mode: fluorophore

Cq determination: single threshold

Baseline method:

FAM: autocalculated

Threshold setting:

FAM: 64.05, autocalculated

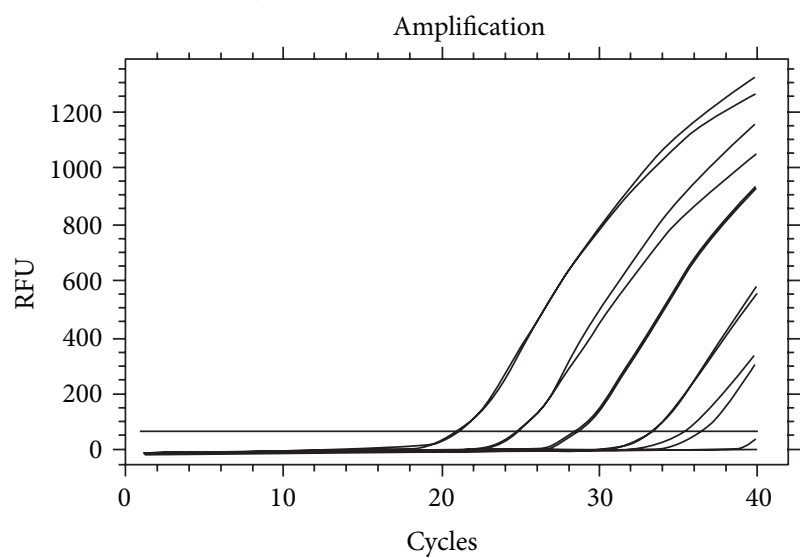

Figure 2: Amplification curve: amplification of the standards (from $10^{6}$ Leish $/ \mathrm{mL}$ to 100 Leish $/ \mathrm{mL}$ ). Below the threshold the nonamplified samples (negative).

\section{Disclosure}

This research received no specific grant from any funding agency in the public, commercial, or not-for-profit sectors.

\section{Conflict of Interests}

All the authors (E. Spada, D. Proverbio, A. Migliazzo, A. Della Pepa, R. Perego, and G. Bagnagatti De Giorgi) declare that they have no conflict of interests.

\section{References}

[1] G. Baneth, "Leishmaniases," in Infectious Diseases of the Dog and Cat, C. E. Greene, Ed., pp. 685-698, Saunders Elsevier, St Louis, Mo, USA, 5th edition, 2006.

[2] R. Gothe, I. Nolte, and W. Kraft, "Leishmaniosis of dogs in Germany: epidemiological case analysis and alternative to conventional causal therapy," Tierarztliche Praxis Ausgabe G, vol. 25, no. 1, pp. 68-73, 1997.

[3] C. Bogdan, G. Schönian, A.-L. Bañuls et al., "Visceral leishmaniasis in a German child who had never entered a known endemic area: case report and review of the literature," Clinical Infectious Diseases, vol. 32, no. 2, pp. 302-306, 2001.

[4] M. M. Díaz-Espińeira and R. J. Slappendel, "A case of autochthonous canine leishmaniasis in the Netherlands," Veterinary Quarterly, vol. 19, no. 2, pp. 69-71, 1997.

[5] P. M. Schantz, F. J. Steurer, Z. H. Duprey et al., "Autochthonous visceral leishmaniasis in dogs in North America," Journal of the American Veterinary Medical Association, vol. 226, no. 8, pp. 1316-1322, 2005.

[6] K. Koehler, M. Stechele, U. Hetzel et al., "Cutaneous leishmaniosis in a horse in southern Germany caused by Leishmania infantum," Veterinary Parasitology, vol. 109, no. 1-2, pp. 9-17, 2002.

[7] L. Lobsiger, N. Müller, T. Schweizer et al., "An autochthonous case of cutaneous bovine leishmaniasis in Switzerland," Veterinary Parasitology, vol. 169, no. 3-4, pp. 408-414, 2010.

[8] C. Ozon, P. Marty, F. Pratlong et al., "Disseminated feline leishmaniosis due to Leishmania infantum in Southern France," Veterinary Parasitology, vol. 75, no. 2-3, pp. 273-277, 1998.

[9] M. G. Pennisi, "A high prevalence of feline leishmaniasis in southern Italy, in Canine Leishmaniasis: Moving Towards a Solution, R. Killick-Kendrick, Ed., pp. 39-48, Intervet International, Boxmeer, The Netherlands, 2002.

[10] A. Poli, F. Abramo, P. Barsotti et al., "Feline leishmaniosis due to Leishmania infantum in Italy," Veterinary Parasitology, vol. 106, no. 3, pp. 181-191, 2002.

[11] M. G. Pennisi, T. Lupo, D. Malara, M. Masucci, A. Migliazzo, and G. Lombardo, "Serological and molecular prevalence of Leishmania infantum infection in cats from southern Italy," Journal of Feline Medicine \& Surgery, vol. 14, pp. 656-657, 2012.

[12] C. Ozon, P. Marty, and A. Lelievre, "Le chat reservoir de Leishmania infantum dans le sud del a France?" in Proceedings of the 24th World Small Animal Veterinary Association Congress, Lyon, France, September 1999.

[13] S. Vita, D. Santori, I. Aguzzi, E. Petrotta, and A. Luciani, "Feline leishmaniasis and ehrlichiosis: serological investigation in Abruzzo region," Veterinary Research Communications, vol. 29, supplement 2, pp. 319-321, 2005.

[14] J. Martín-Sánchez, C. Acedo, M. Muñoz-Pérez, B. Pesson, O. Marchal, and F. Morillas-Márquez, "Infection by Leishmania infantum in cats: epidemiological study in Spain," Veterinary Parasitology, vol. 145, pp. 267-273, 2007.

[15] L. Solano-Gallego, A. Rodríguez-Cortés, L. Iniesta et al., "Crosssectional serosurvey of feline leishmaniasis in ecoregions around the Northwestern Mediterranean," American Journal of Tropical Medicine and Hygiene, vol. 76, no. 4, pp. 676-680, 2007.

[16] T. Ayllon, M. A. Tesouro, I. Amusategui, A. Villaescusa, F. Rodriguez-Franco, and Á. Sainz, "Serologic and molecular evaluation of Leishmania infantum in cats from central Spain," Annals of the New York Academy of Sciences, vol. 1149, pp. 361364, 2008. 
[17] A. Diakou, E. Papadopoulos, and K. Lazarides, "Specific antiLeishmania spp. antibodies in stray cats in Greece," Journal of Feline Medicine and Surgery, vol. 11, no. 8, pp. 728-730, 2009.

[18] L. Cardoso, A. P. Lopes, K. Sherry, H. Schallig, and L. SolanoGallego, "Low seroprevalence of Leishmania infantum infection in cats from northern Portugal based on DAT and ELISA," Veterinary Parasitology, vol. 174, no. 1-2, pp. 37-42, 2010.

[19] A. Duarte, I. Castro, I. M. Pereira da Fonseca et al., "Survey of infectious and parasitic diseases in stray cats at the Lisbon Metropolitan Area, Portugal," Journal of Feline Medicine and Surgery, vol. 12, no. 6, pp. 441-446, 2010.

[20] C. Maia, J. Gomes, J. Cristóvão et al., "Feline Leishmania infection in a canine leishmaniasis endemic region, Portugal," Veterinary Parasitology, vol. 174, no. 3-4, pp. 336-340, 2010.

[21] T. Ayllón, P. P. V. P. Diniz, E. B. Breitschwerdt, A. Villaescusa, F. Rodríguez-Franco, and A. Sainz, "Vector-borne diseases in client-owned and stray cats from Madrid, Spain," Vector-Borne and Zoonotic Diseases, vol. 12, no. 2, pp. 143-150, 2012.

[22] E. Spada, D. Proverbio, A. Della Pepa et al., "Seroprevalence of feline immunodeficiency virus, feline leukaemia virus and Toxoplasma gondii in stray cat colonies in northern Italy and correlation with clinical and laboratory data," Journal of Feline Medicine \& Surgery, vol. 14, pp. 369-377, 2012.

[23] World Organization for Animal Health (OIE), "Manual of Diagnostic Tests and Vaccines for Terrestrial Animals," CAP. 2.1.8, section B, paragraph 2, 2008, http://www.oie.int/fileadmin/Home/eng/Health_standards/tahm/2.01.08_LEISHMANIOSIS.pdf.

[24] M.-D. Tabar, L. Altet, O. Francino, A. Sánchez, L. Ferrer, and X. Roura, "Vector-borne infections in cats: molecular study in Barcelona area (Spain)," Veterinary Parasitology, vol. 151, no. 24, pp. 332-336, 2008.

[25] M. Maroli, M. G. Pennisi, T. Di Muccio, C. Khoury, L. Gradoni, and M. Gramiccia, "Infection of sandflies by a cat naturally infected with Leishmania infantum," Veterinary Parasitology, vol. 145, no. 3-4, pp. 357-360, 2007.

[26] S. Reale, L. Maxia, F. Vitale, N. S. Glorioso, S. Caracappa, and G. Vesco, "Detection of Leishmania infantum in dogs by PCR with lymph node aspirates and blood," Journal of Clinical Microbiology, vol. 37, no. 9, pp. 2931-2935, 1999.

[27] E. Spada, D. Proverbio, C. Giudice, M. DiGiancamillo, M. Lodi, and R. Perego, "Pituitary-dependent hyperadrenocorticism and generalised toxoplasmosis in a cat with neurological signs," Journal of Feline Medicine and Surgery, vol. 12, no. 8, pp. 654$658,2010$.

[28] M. Maroli, L. Rossi, R. Baldelli et al., "The northward spread of leishmaniasis in Italy: evidence from retrospective and ongoing studies on the canine reservoir and phlebotomine vectors," Tropical Medicine and International Health, vol. 13, no. 2, pp. 256-264, 2008.

[29] E. Spada and D. Proverbio, "Canine leishmaniasis in nonendemic area: incidence of positivity to IFAT test in dogs moved to endemic area," in Proocedings of the LVI National Congress SISVET, pp. 289-290, Giardini Naxos, Italy, September 2002.

[30] E. Spada, D. Proverbio, D. Groppetti, R. Perego, V. Grieco, and E. Ferro, "First report of the use of meglumine antimoniate for treatment of canine leishmaniasis in a pregnant dog," Journal of the American Animal Hospital Association, vol. 47, no. 1, pp. 67-71, 2011.

[31] R. Perego, D. Proverbio, E. Spada, and E. Ferro, "Canine leishmaniasis: a sero-epidemiological survey by indirect fluorescence antibody test (IFAT) in 313 dogs at sanitary public kennel of Milan," in Proceedings of the LIX Congress SISVET, pp. 273-274, Viareggio, Italy, September 2005.

[32] European Scientific Counsel Companion Animal Parasites (ESCCAP), "Control of Vector-Borne Diseases in Dogs and Cats," Guideline 05, 2nd edition, October 2012, http:// www.esccap.org/.

[33] E. D. C. Ferreira, M. de Lana, M. Carneiro et al., "Comparison of serological assays for the diagnosis of canine visceral leishmaniasis in animals presenting different clinical manifestations," Veterinary Parasitology, vol. 146, no. 3-4, pp. 235-241, 2007. 

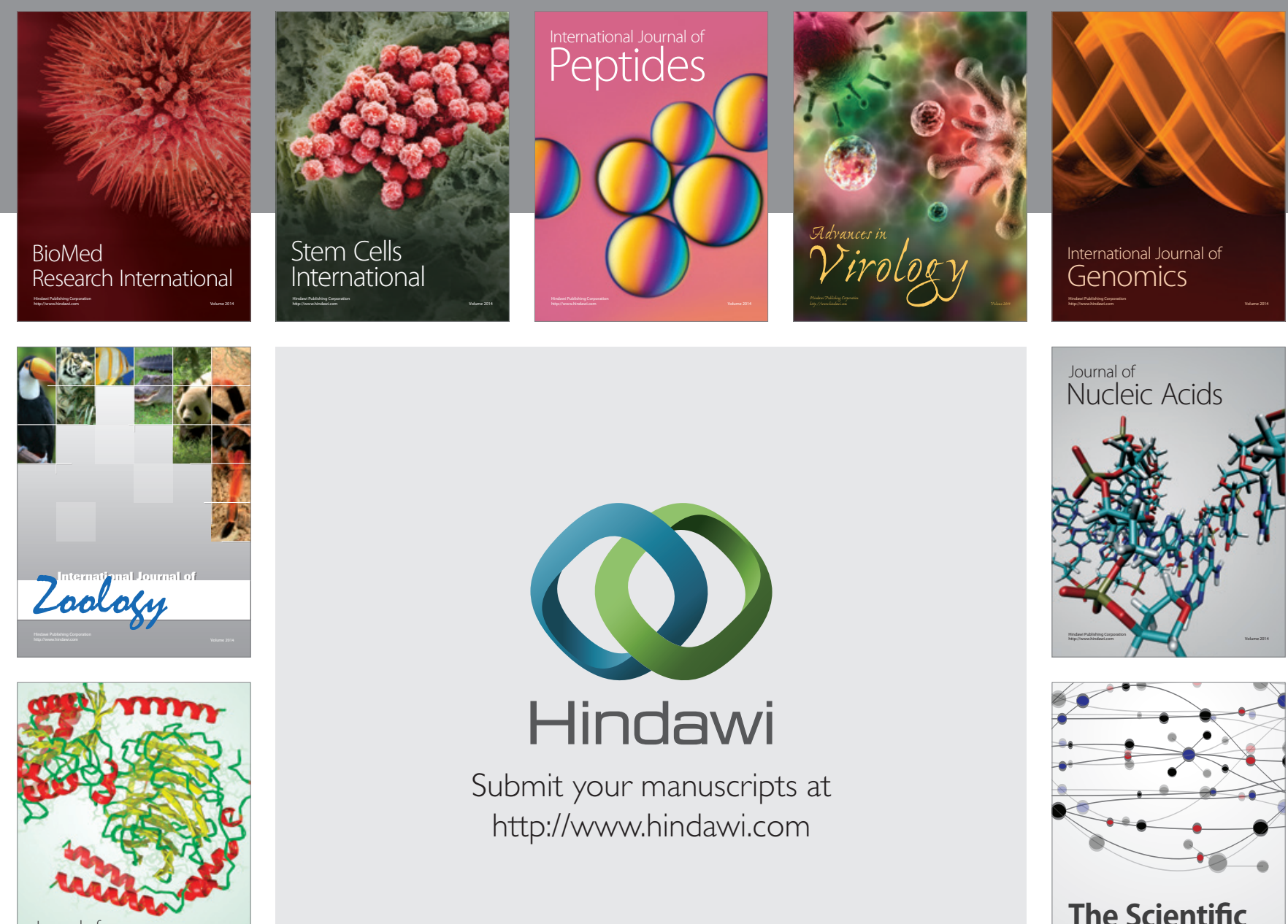

Submit your manuscripts at

http://www.hindawi.com

Journal of
Signal Transduction
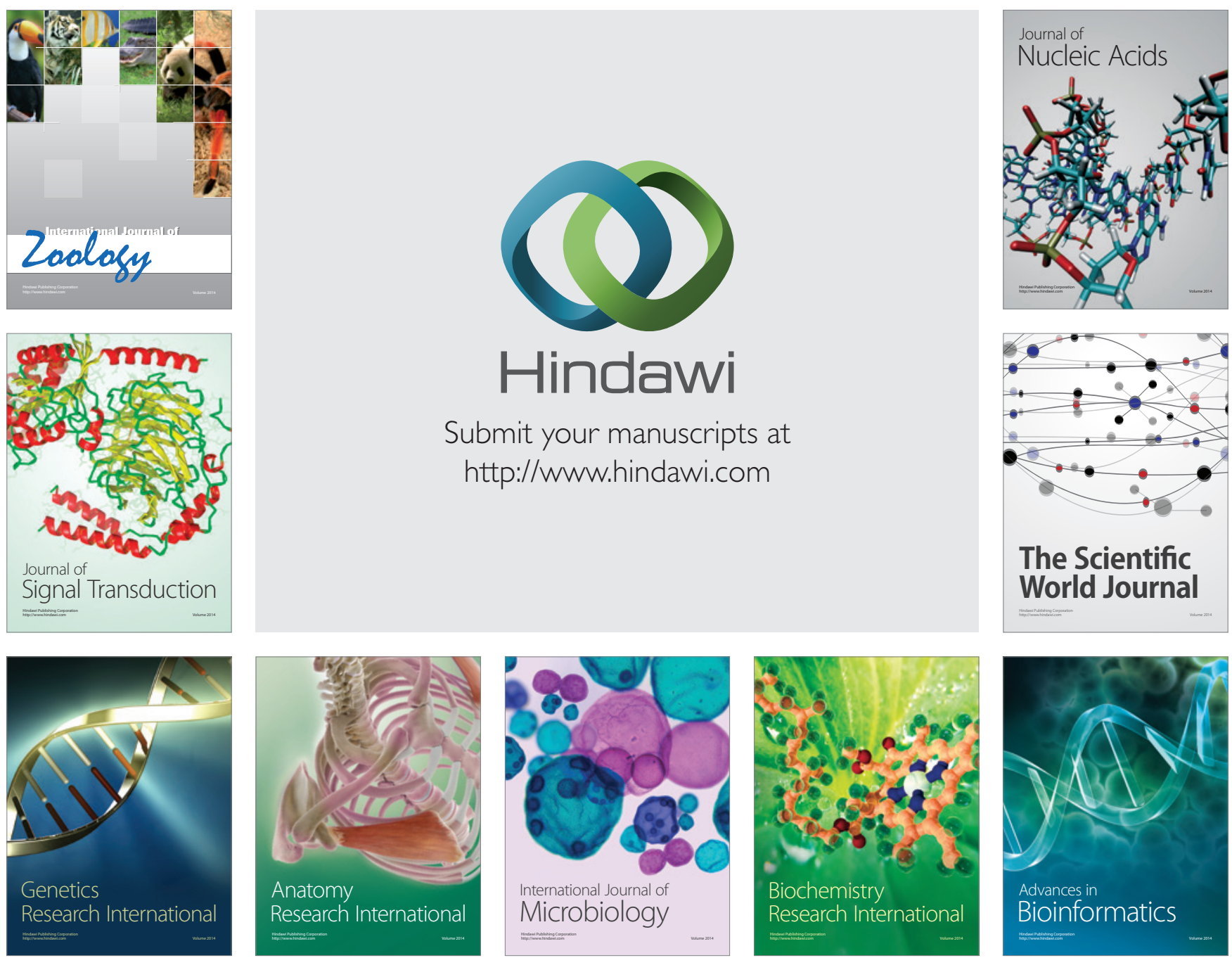

The Scientific World Journal
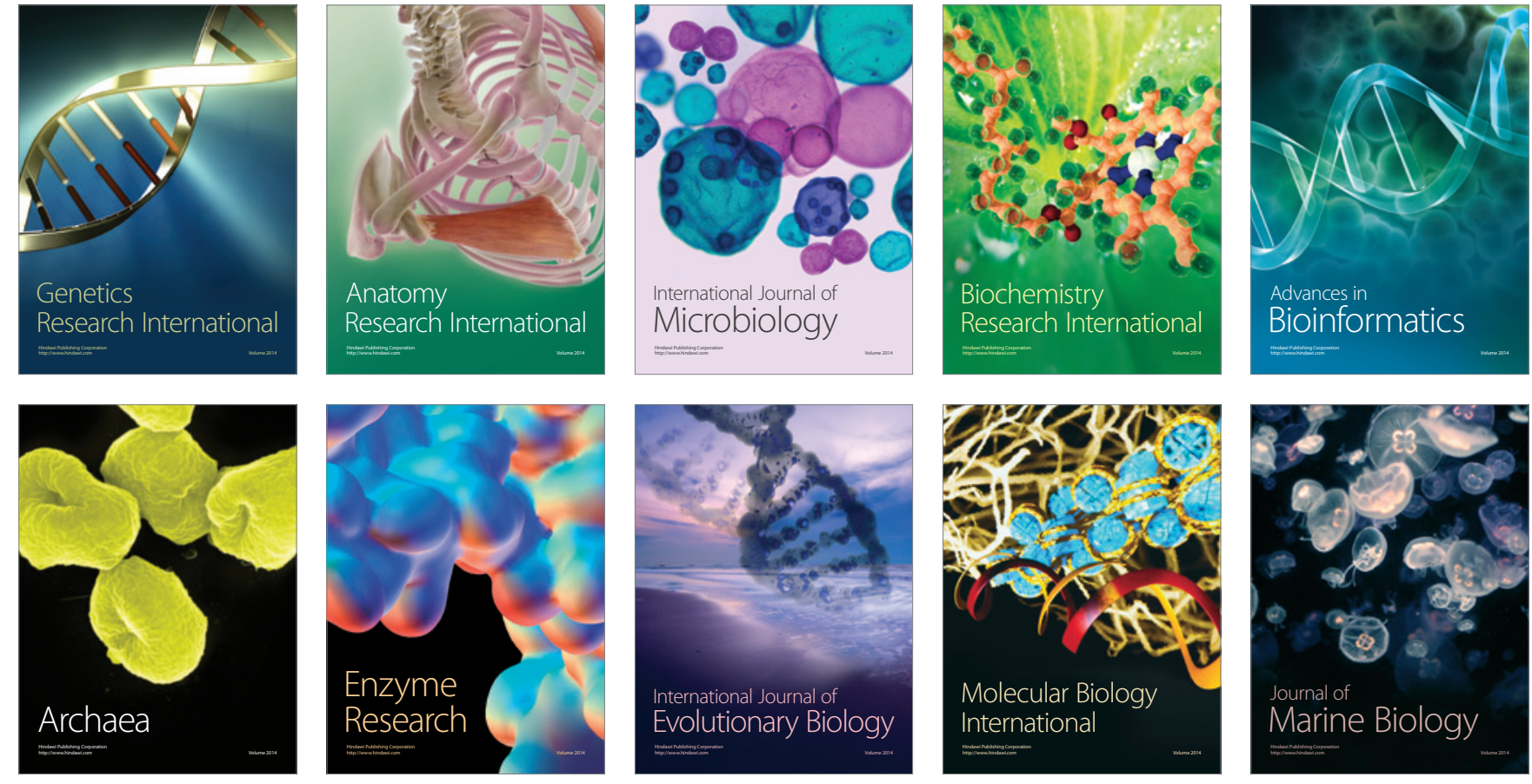